\title{
Analysis of a Risky Two Unit System under Marked Process Incorporating Two Repairmen with Vacations
}

\author{
Nidhi Tiwari \\ Department of Applied Sciences, Teerthanker Mahaveer University, \\ Moradabad, U. P. 244001, India \\ E-mail: nidhitiwari26@gmail.com
}

\author{
S. B. Singh \\ Department of Mathematics, Statistics and Computer Science, G. B. Pant University of Agriculture and Technology, \\ Pantnagar, Uttarakhand, 263145, India \\ E-mail: drsurajbsingh@yahoo.com
}

Received 26 June 2015

Accepted 10 November 2015

\begin{abstract}
In this paper the system considered consists of two subsystems $\mathrm{A}$ and $\mathrm{B}$. Subsystem $\mathrm{A}$ has only one unit whereas subsystem $B$ consists of two homogeneous units $B_{1}$ and $B_{2}$. Here $B_{2}$ is in hot standby with $B_{1}$. In the present study we have incorporated two repairmen namely supervisor and novice to repair the failed units of the system. The supervisor is always there whereas the novice remains in vacation. The system is analyzed under "Preemptiveresume-repair discipline". We have used supplementary variable technique, Laplace transformation and copula to obtain various transition state probabilities, reliability, availability, cost analysis and the steady state behaviour of the system. To make a comparison between the measures obtained using two families of copulas Gumbel-Hougaard family and Bivariate Clayton, the model has been solved with the help of Gumbel-Hougaard family of copula and Bivariate Clayton copula both in two different cases. Also a comparison between two types of repair policies "Head-of-line-repair" and "Preemptive-resume-repair" has been carried out. At last some numerical examples have been taken.
\end{abstract}

Keywords: Reliability, Marked process, cost analysis, Gumbel-Hougaard copula, Bivariate Clayton copula, supplementary variable technique.

\section{Introduction}

Recent advances in science and technology have led to a rapid increase in the complexity of most engineered systems. These complex engineered systems are composed of many subsystems due to which it is very risky to use them and they are characterized by observable complex behaviour. Data from these repairable systems generally contain more information than only failure time. To find the reliability of such complex repairable system going to be a central challenge for engineers, mathematicians and statisticians. Marked process (Lindqvist and Bedford,
2004) can prove to be more useful technique to tackle this problem. It is a well known phenomenon that to increase the reliability and availability of the system a unit that has failed is repaired. The repair facility may have a single repairman or more than one repairman to repair the failed units. In last decades there has been a growing interest among researchers (Gupta and Agarwal, 1984; Ram and Singh, 2010) in the reliability analysis of complex system incorporating one repairman. Of course there are researchers (Garg and Goel, 1985; Gupta and Sharma, 1993; Goel and Gupta, 1985) who modeled the complex system incorporating two repairmen or two repair facilities or two repair 
distributions. But they did not include the vacations of repairman in their study. Though there are good studies (Linmin and Jiandong, 2009; Tuteja and Malik, 1992) who have given attention to the vacations of repairman but their modeling includes only one repairman. Furthermore a review of past work (Kovalenko and Smolich, 2001) shows that while analyzing complex systems there can be two situations: (i) repairman is a full time employee of the organization (ii) due to present day condition in mind managerial staff of a company has to face tough time which becomes especially aggravating during the current economic crisis leading to appointment of employees not on permanent basis. Both these situations lead to vacations of repairman, who will be called for repairing as and when they are required. Hence vacation of the repairman can also play key role in the analysis of repairable complex systems.

This paper is an extension of the work done in our two previous papers (Tiwari and Singh, 2010; Tiwari and Singh, 2010) in which we have used "Head-of-linerepair" and "Preemptive-repeat-repair" policies to repair the failed units. With above facts in view the present paper examines and proposes an approach to deal with the complex system incorporating information gathered due to marked process having two repairmen with different skills and availability. The system under study consists of two subsystems A and B arranged in series configuration. A has only one unit while $\mathrm{B}$ consists of two identical units $B_{1}$ and $B_{2}$ (Demcese, 2009; Kumar and Singh, 2008). The failure rates of both the units of subsystem B are same. Subsystem A has two states: good and failed, while B can be in any of the three states: good, degraded and failed. Two types of failure viz. partial and catastrophic can occur in subsystem $B$. In case of partial failure subsystem B goes in the state of partial failure which brings the whole system to the state of reduced efficiency. While in case of catastrophic failure the subsystem B and hence the whole system fails completely. The system can further fail completely if (i) subsystem A fails or (ii) both the units of subsystem B fail. There are two repairmen namely supervisor and novice available to repair the failed units of the system. The novice is not a regular worker he remains in vacation and can be called for repairing as per the requirements. Further, it is assumed that novice can repair only subsystem $\mathrm{B}$ and he is not good at repairing of subsystem A. Also, whenever there is a failure in a unit of subsystem B, supervisor starts repairing it but if at the same time there occurs any failure in other unit of B then novice is called for repairing. The system is under "Preemptive-resumerepair discipline" where subsystem A has given priority over subsystem B. The present system is under marked process so at any state more than one type of failure can take place. The two types of failures between two states can be calculated with the help of copula (Nelson, 2006; Yan Jun, 2007). Here in the present study it is assumed that the failure from state $S_{0}$ to $S_{2}$ follows two distributions, i.e. constant and logarithmic. System can be repaired in two ways from states $S_{6} / S_{2}$ to $S_{0}$ as well. Failure rates are assumed to be constant in general whereas the repairs done by supervisor and novice follow general and exponential distribution respectively. The research is part of larger effort to investigate the application of copula methodology for reliability analysis into the decision making process. The model has been solved by using two different types of copulas viz. Gumbel-Hougaard and Bivariate Clayton. The following characteristics of the system have been analyzed:

1. Transition state probabilities of the system.

2. Asymptotic behaviour of the system.

3. Various measures such as reliability, availability, M.T.T.F. analysis and cost effectiveness of the system.

The following comparative studies have also been made to test the applicability of repair policies and copulas during the analysis of model:

(1) A comparison between availabilities of two different types of repair policies, namely "Head-ofline-repair" and "Preemptive-resume-repair" with the application of copula.

(2) A comparison between the various measures of reliability when two different copulas, i.e. GumbelHougaard and Bivariate Clayton are applied.

\subsection{Copula}

A two dimensional copula is a function $\mathrm{C}:[0,1] \times[0,1]$ $\rightarrow[0,1]$ that satisfies following two properties.

1. Boundary conditions:

(a) For all $\mathrm{t}$ in $[0,1], \mathrm{C}(\mathrm{t}, 0)=\mathrm{C}(0, \mathrm{t})=0$

(b) For all $\mathrm{t}$ in $[0,1], \mathrm{C}(\mathrm{t}, 1)=\mathrm{C}(1, \mathrm{t})=\mathrm{t}$

2. Rectangular inequality: If $\mathrm{u}_{1}, \mathrm{u}_{2}, \mathrm{v}_{1}, \mathrm{v}_{2}$ are in $[0,1]$ with $\mathrm{u}_{1} \leq \mathrm{u}_{2}$ and $\mathrm{v}_{1} \leq \mathrm{v}_{2}$, then

$\mathrm{C}\left(\mathrm{u}_{2}, \mathrm{v}_{2}\right)-\mathrm{C}\left(\mathrm{u}_{1}, \mathrm{v}_{2}\right)-\mathrm{C}\left(\mathrm{u}_{2}, \mathrm{v}_{1}\right)+\mathrm{C}\left(\mathrm{u}_{1}, \mathrm{v}_{1}\right) \geq 0$ 


\subsubsection{Bivariate Clayton copula}

A two dimensional Bivariate Clayton copula is defined as

$$
C_{\theta}\left(u_{1}, u_{2}\right)=\left(u_{1}^{-\theta}+u_{2}^{-\theta}-1\right)^{-1 / \theta}
$$

The Clayton copula is well defined for $0<\theta<\infty$ and for $\theta \rightarrow 0$ and $\theta \rightarrow \infty$ it converges to the product copula and comonotonicity respectively. It is not symmetric and has lower tail dependence (no upper tail dependence). Due to the property of lower tail dependence, the Clayton copula is a possible candidate for model building specially in the financial context.

\subsubsection{Bivariate Gumbel-Hougaard family copula}

A two dimensional Bivariate Clayton copula is defined as $C_{\theta}\left(u_{1}, u_{2}\right)=\exp \left(-\left(\left(-\log u_{1}\right)^{\theta}+\left(-\log u_{2}\right)^{\theta}\right)^{1 / \theta}\right), 1 \leq \theta \leq \infty$ For $\theta=1$ the Gumbel-Hougaard copula models independence, for $\theta \rightarrow \infty$ it converges to comonotonicity. It is not symmetric and has upper tail dependence (no lower tail dependence) also it has positive dependence.

\subsubsection{Applications of copula in the present study}

Copula representations of multivariate distributions allow us to fit any marginal we like to different random variables, and these distributions might differ from one variable to another. In the present paper we have applied copula to find the joint probability distribution of repair/failure probabilities following different types of distribution.

\subsection{Repair policies}

The comparison between two repair policies has been made in the present study: "Head-of line- repair" and "Preemptive-resume-repair".

\subsubsection{Head-of-line-repair}

In this repair policy the failed unit will be repair according to first come first get. In state $S_{3}$ when the system is already in degraded state due to the failure in one of the unit of subsystem B if subsystem A also fails, it comes to state $\mathrm{S}_{4}$ which is a fully failed state. In Head-of-line-repair policy firstly the repair of failed B unit will be completed after that failed A unit will be repair.

\subsubsection{Preemptive-resume-repair}

In this type of repair we give priority to one unit over other unit for repair. In the present model we have given priority to Subsystem A over B. So when the system comes to state $\mathrm{S}_{4}$ in which one of subsystem $B$ unit has already in repair in $\mathrm{S}_{3}$ state and now A unit also fails, according to this policy we have given priority to Subsystem A over B so the repairman will start to repair subsystem A. After the repair of subsystem A he will start to repair subsystem B from the point where he has left it.

\section{Assumptions}

(1) Initially the system is in good state.

(2) Subsystems A and B are connected in series.

(3) Subsystem A has constant failure rate and can be in two states: good and failed.

(4) Subsystem B has three states namely good, degraded and failed.

(5) System is under marked process.

(6) Repairs done by the supervisor and novice follow general time distribution and exponential time distribution respectively.

(7) There are two types of failures from state $S_{0}$ to $S_{2}$ one is constant and other is logarithmic.

(8) There are two types of repair between $\mathrm{S}_{6} / \mathrm{S}_{2}$ to $\mathrm{S}_{0}$.

(9) Subsystem A can be repaired by supervisor only.

(10) System is under preemptive resume repair discipline, i. e. the preempted B is taken back in repair facility after priority repair of A from the point where it has been left.

(11) After repair the system is as good as new.

(12) In case of catastrophic failure both the repairmen repair the system.

(13) Joint probability distribution of repair rates, where repair is done by supervisor and novice follows Gumbel-Hougaard family and Bivariate Clayton copula.

\section{State specification}

Table to mention the different states of the system, the block diagram of the system and transition state diagram of the system are given in next page.

Here we have used some notations as

$$
\begin{aligned}
& \mathrm{G}=\text { Good state, } \\
& F=\text { Failed state, } \\
& D=\text { Degraded state, }
\end{aligned}
$$


$\mathrm{F}_{\mathrm{R}}=$ Failed under repair,

$\mathrm{D}_{\mathrm{R}}=$ Degraded state under repair,

$\mathrm{F}_{\mathrm{Rv}}=$ Failed state, one unit is under repair and novice is in vacation.

Table 1: State specification chart

\begin{tabular}{|c|c|c|c|}
\hline States & $\begin{array}{l}\text { State of } \\
\text { subsystem A }\end{array}$ & $\begin{array}{l}\text { Subsystem B: } \\
\text { number of good } \\
\text { units }\end{array}$ & $\begin{array}{l}\text { System } \\
\text { state }\end{array}$ \\
\hline $\mathrm{S}_{0}$ & $\mathrm{G}$ & 2 & G \\
\hline $\mathrm{S}_{1}$ & $\mathrm{~F}$ & 2 & $\mathrm{~F}_{\mathrm{R}}$ \\
\hline $\mathrm{S}_{2}$ & G & 0 & $F_{R}$ \\
\hline $\mathrm{S}_{3}$ & $\mathrm{G}$ & 1 & $D_{R}$ \\
\hline $\mathrm{S}_{4}$ & $\mathrm{~F}$ & 1 & $\mathrm{~F}_{\mathrm{R}}$ \\
\hline $\mathrm{S}_{5}$ & G & 0 & $\mathrm{~F}_{\mathrm{Rv}}$ \\
\hline $\mathrm{S}_{6}$ & $\mathrm{G}$ & 0 & $F_{R}$ \\
\hline
\end{tabular}

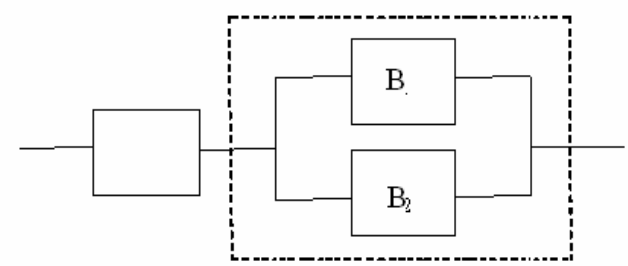

Fig. 1: Block diagram of the system

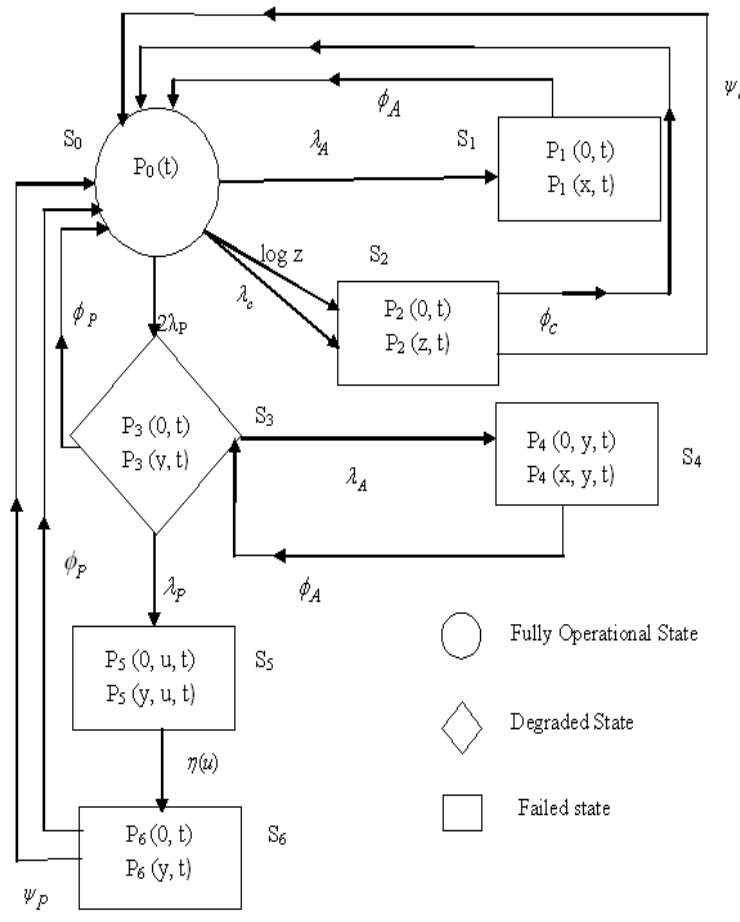

Fig 2: Transition state diagram

\section{Notations}

$\lambda_{A} \quad$ Failure rate of subsystem A.
$\lambda_{P}, \lambda_{C} \quad$ Failure rates of subsystem B corresponding to partial and catastrophic failures for both the units.

$\phi_{i}(r) \quad$ Repair rate of supervisor.

$\psi_{i}(r) \quad$ Repair rate of novice if $\mathrm{i}=\mathrm{A} / \mathrm{B} / \mathrm{C}$ then $\mathrm{r}=$ $\mathrm{x} / \mathrm{y} / \mathrm{z}$ respectively.

$\mathrm{x} \quad$ Elapsed repair time for the subsystem A.

$\mathrm{y}, \mathrm{z} \quad$ Elapsed repair times for the partial and catastrophic failures respectively for the subsystem B.

$\eta, \mathrm{u} \quad$ Vacation rate and variable for vacation of repairman.

$\mathrm{P}_{\mathrm{i}}(\mathrm{t}) \quad$ Probability that the system is in $\mathrm{S}_{\mathrm{i}}$ state at an instant $\mathrm{t}$ for $\mathrm{i}=1,2, \ldots, 6$.

$\overline{\mathrm{P}}_{\mathrm{i}}(\mathrm{s}) \quad$ Laplace transform of $\mathrm{P}_{\mathrm{i}}(\mathrm{t})$.

$\mathrm{P}_{4}(\mathrm{y}, \mathrm{t}) \quad$ Probability density function that at time $\mathrm{t}$ the system is in failed state $\mathrm{S}_{4}$ and the system is under repair, elapsed repair time is $\mathrm{y}$.

$\mathrm{E}_{\mathrm{p}}(\mathrm{t}) \quad$ Expected profit during the interval $(0, \mathrm{t}]$.

$\mathrm{K}_{1}, \mathrm{~K}_{2} \quad$ Revenue per unit time and service cost per unit time respectively.

$\mathrm{S}_{\eta}(\mathrm{x}) \quad \eta(\mathrm{x}) \exp \left(-\int_{0}^{\mathrm{x}} \eta(\mathrm{x}) \mathrm{dx}\right)$

$\overline{\mathrm{S}}_{\eta}(x) \quad$ Laplace transform of $\mathrm{S}_{\eta}(\mathrm{x})$

$S_{\eta}(x)=\int_{0}^{\infty} \eta(x) \exp \left(-s x-\int_{0}^{x} \eta(x) d x\right) d x$

If $u_{1}=\phi_{P}(y), \quad u_{2}=\psi_{P}(y)$ then the expression for the joint probability according to:

(i) Gumbel-Hougaard family of copula is given as

$C_{\theta}\left(u_{1}, u_{2}\right)=\exp \left[-\left\{\left(-\log \phi_{P}(y)\right)^{\theta}+\left(-\log \psi_{P}(y)\right)^{\theta}\right\}^{1 / \theta}\right]$

(ii) Bivariate Clayton family of copula is given as $C_{\theta}\left(u_{1}, u_{2}\right)=\left[\left\{\phi_{P}(y)\right\}^{-\theta}+\left(\psi_{P}(y)\right\}^{-\theta}\right]^{-1 / \theta}$

\section{Formulation of Mathematical Model}

By probability considerations and continuity arguments, we obtain the following set of integro-differential equations governing the behaviour of the system.

$$
\begin{aligned}
& {\left[\frac{d}{d t}+\lambda_{A}+\lambda+2 \lambda_{P}\right] P_{0}(t)=\int_{0}^{\infty} \phi_{A}(x) P_{1}(x, t) d x} \\
& +\int_{0}^{\infty} \psi(z) P_{2}(z, t) d z+\int_{0}^{\infty} \psi(y) P_{6}(y, t) d y \\
& +\int_{0}^{\infty} \phi_{P}(y) P_{3}(y, t) d y
\end{aligned}
$$




$$
\begin{aligned}
& {\left[\frac{\partial}{\partial t}+\frac{\partial}{\partial x}+\phi_{A}(x)\right] P_{1}(x, t)=0} \\
& {\left[\frac{\partial}{\partial t}+\frac{\partial}{\partial z}+\psi(z)\right] P_{2}(z, t)=0} \\
& {\left[\frac{\partial}{\partial t}+\frac{\partial}{\partial y}+\lambda_{A}+\lambda_{P}+\phi_{P}(y)\right] P_{3}(y, t)} \\
& \quad=\int_{0}^{\infty} \phi_{A}(x) P_{4}(x, y, t) d x \\
& {\left[\frac{\partial}{\partial t}+\frac{\partial}{\partial x}+\phi_{A}(x)\right] P_{4}(x, y, t)=0} \\
& {\left[\frac{\partial}{\partial t}+\frac{\partial}{\partial y}+\eta(u)\right] P_{5}(y, u, t)=0} \\
& {\left[\frac{\partial}{\partial t}+\frac{\partial}{\partial y}+\psi(y)\right] P_{6}(y, t)=0}
\end{aligned}
$$

\subsection{Boundary conditions}

$$
\begin{aligned}
& P_{1}(0, t)=\lambda_{A} P_{0}(t) \\
& P_{2}(0, t)=\lambda P_{0}(t) \\
& P_{3}(0, t)=2 \lambda_{P} P_{0}(t) \\
& P_{4}(0, y, t)=\lambda_{A} P_{3}(\mathrm{y}, \mathrm{t}) \\
& P_{5}(0, u, t)=\lambda_{P} P_{3}(y, t) \\
& P_{6}(0, t)=\eta(u) P_{5}(y, u, t)
\end{aligned}
$$

\subsection{Initial condition:}

$$
P_{0}(0)=0
$$

and other state probabilities are zero at $\mathrm{t}=0$.

The present model has been studied under two different copulas viz. Gumbel-Hougaard and Bivariate Clayton.

(i) When Gumbel-Hougaard copula is applied to analyse the model then in the equations (1), (3), (7) and (9), $\psi(\mathrm{y}), \psi(\mathrm{z}), \lambda$ and $\xi$ will have the following expressions:

$\psi(y)=\exp \left[-\left\{\left(-\log \psi_{P}(y)\right)^{\theta}+\left(-\log \phi_{p}(y)\right)^{\theta}\right\}^{1 / \theta}\right]$

$\psi(z)=\exp \left[-\left\{\left(-\log \psi_{c}(z)\right)^{\theta}+\left(-\log \phi_{c}(z)\right)^{\theta}\right\}^{1 / \theta}\right.$

$\lambda=\exp \left[-\left\{\left(-\log \lambda_{c}\right)^{\theta}+\left(-\log (\log z)^{\theta}\right\}^{1 / \theta}\right.\right.$

$\xi(y)=\phi_{p}(y)+\psi(y)$

(ii) When Bivariate-Clayton copula is applied to analyse the model then in the equations (1), (3), (7) and (9), $\psi(\mathrm{y}), \psi(\mathrm{z}), \lambda$ and $\xi$ will have the following expressions:

$$
\begin{aligned}
& \psi(y)=\left[\left\{\psi_{p}(y)\right\}^{-\theta}+\left\{\phi_{p}(y)\right\}^{-\theta}\right]^{-1 / \theta} \\
& \psi(z)=\left[\left\{\psi_{c}(z)\right\}^{-\theta}+\left\{\phi_{c}(z)\right\}^{-\theta}\right]^{-1 / \theta} \\
& \lambda=\left[\left(\lambda_{c}\right)^{-\theta}+(\log z)^{-\theta}\right]^{-1 / \theta} \\
& \xi(y)=\phi_{p}(y)+\psi(y)
\end{aligned}
$$

\section{Solution of the model}

Taking Laplace transformation of equations (1-13) and using (14), we get

$$
\begin{aligned}
& {\left[s+\lambda_{A}+\lambda+2 \lambda_{P} \bar{P}_{0}(s)=1+\int_{0}^{\infty} \phi_{A}(x) \bar{P}_{1}(x, s) d x\right.} \\
& +\int_{0}^{\infty} \psi(z) \bar{P}_{2}(z, s) d z+\int_{0}^{\infty} \psi(y) \bar{P}_{6}(y, s) d y \\
& \quad+\int_{0}^{\infty} \phi_{P}(y) \bar{P}_{3}(y, s) d y \\
& {\left[s+\frac{\partial}{\partial x}+\phi_{A}(x)\right] \bar{P}_{1}(x, s)=0} \\
& {\left[s+\frac{\partial}{\partial z}+\psi(z)\right] \bar{P}_{2}(z, s)=0} \\
& {\left[s+\frac{\partial}{\partial y}+\lambda_{A}+\lambda_{P}+\phi_{P}(y)\right] \bar{P}_{3}(y, s)} \\
& \quad=\int_{0}^{\infty} \phi_{A}(x) \bar{P}_{4}(x, y, s) d x \\
& {\left[s+\frac{\partial}{\partial x}+\phi_{A}(x)\right] \bar{P}_{4}(x, y, s)=0} \\
& {\left[s+\frac{\partial}{\partial y}+\eta(u)\right] \bar{P}_{s}(y, u, s)=0} \\
& \left.\left[s+\frac{\partial}{\partial y}+\psi(y)\right]\right] \bar{P}_{6}(y, s)=0
\end{aligned}
$$

6.1 Boundary conditions

$$
\begin{aligned}
& \bar{P}_{1}(0, s)=\lambda_{A} \bar{P}_{0}(\mathrm{~s}) \\
& \bar{P}_{2}(0, s)=\lambda \bar{P}_{0}(\mathrm{~s}) \\
& \bar{P}_{3}(0, s)=2 \lambda_{P} \bar{P}_{0}(s) \\
& \bar{P}_{4}(0, y, s)=\lambda_{A} \bar{P}_{3}(\mathrm{y}, \mathrm{s}) \\
& \bar{P}_{5}(0, u, s)=\lambda_{P} \bar{P}_{3}(y, s) \\
& \bar{P}_{6}(0, s)=\eta(u) \bar{P}_{5}(y, u, s)
\end{aligned}
$$

Solving equations (15-21) and using equations (2227), one can get the following transition state probabilities 


$$
\begin{aligned}
& \bar{P}_{0}(s)=\frac{1}{D(s)} \\
& \bar{P}_{1}(s)=\lambda_{A}\left[\frac{1-\bar{S}_{\phi_{A}}(s)}{s}\right] \bar{P}_{0}(s) \\
& \bar{P}_{2}(s)=\lambda\left[\frac{1-\bar{S}_{\psi_{z}}(s)}{s}\right] \bar{P}_{0}(s) \\
& \bar{P}_{3}(s)=2 \lambda_{P} \bar{P}_{0}(s)\left[\frac{1-\bar{S}_{\phi_{P}}\left(s^{*}\right)}{s^{*}}\right] \\
& \bar{P}_{4}(s)=2 \lambda_{A} \lambda_{P}\left[\frac{1-\bar{S}_{\phi_{A}}(s)}{s}\right] \\
& {\left[\frac{1-\bar{S}_{\phi P}\left(s^{*}\right)}{s *}\right] \bar{P}_{0}(s)} \\
& \bar{P}_{s}(s)=2 \lambda_{P}{ }^{2}\left[\frac{1-\bar{S}_{\phi P}\left(s+s^{*}+\eta(u)\right)}{s+s^{*}+\eta(u)}\right] \bar{P}_{0}(s) \\
& \bar{P}_{6}(s)=\left[\frac{1-\bar{S}_{\xi}\left(2 s+s^{*}+\eta(u)\right)}{2 s+s^{*}+\eta(u)}\right] \\
& \eta(u) 2 \lambda_{p}{ }^{2} \bar{P}_{0}(s)
\end{aligned}
$$

where

$$
\begin{aligned}
& s^{*}=s+\lambda_{A}+\lambda_{P}-\lambda_{A} \bar{S}_{\phi_{A}}(s) \\
& D(s)=s+\lambda_{A}+\lambda+2 \lambda_{P}-\lambda_{A} \bar{S}_{\phi_{A}}(s) \\
& -2 \lambda_{P} \bar{S}_{\phi_{P}}\left(s^{*}\right)-\lambda \bar{S}_{\psi_{z}}(s)-2 \lambda_{P}^{2} \eta(u) \\
& \int_{0}^{\infty} \psi(y) \exp \left\{-\left(2 s+s^{*}+\eta(u)\right) y-\int_{0}^{y} \xi(y) d y\right\}
\end{aligned}
$$

Also up and down state probabilities of the system is given by

$$
\begin{aligned}
\bar{P}_{\text {up }}(s)= & \bar{P}_{0}(s)+\bar{P}_{3}(s) \\
= & \left\{1+2 \lambda_{P}\left[\frac{1-\bar{S}_{\phi P}\left(s^{*}\right)}{s *}\right]\right\} \bar{P}_{0}(s) \\
\bar{P}_{\text {down }}(s)= & \bar{P}_{1}(s)+\bar{P}_{2}(s)+\bar{P}_{4}(s) \\
& +\bar{P}_{s}(s)+\bar{P}_{6}(s)
\end{aligned}
$$

$$
\begin{aligned}
& =\left[\lambda_{\mathrm{A}}\left\{\frac{1-\bar{S}_{\phi_{A}}(s)}{s}\right\}+\lambda\left\{\frac{1-\bar{S}_{\psi_{z}}(s)}{s}\right\}\right] \bar{P}_{0}(s) \\
& +2 \lambda_{A} \lambda_{P}\left\{\frac{1-\bar{S}_{\phi_{A}}(s)}{s}\right\} \times\left\{\frac{1-\bar{S}_{\phi_{P}}\left(s^{*}\right)}{s^{*}}\right\} \bar{P}_{0}(s) \\
& +2 \lambda_{P}{ }^{2} \bar{P}_{0}(s)\left[\frac{1-\bar{S}_{\phi_{P}}(s+s *+\eta(u))}{s+s^{*}+\eta(u)}\right]+2 \eta(u) \lambda_{P}{ }^{2} \\
& \quad \times \bar{P}_{0}(s) \times\left[\frac{1-\bar{S}_{\xi}(2 s+s *+\eta(u))}{2 s+s^{*}+\eta(u)}\right]
\end{aligned}
$$

Also it is noticeable that

$$
\bar{P}_{\text {up }}(s)+\bar{P}_{\text {down }}(s)=1 / s
$$

The present model has been studied under two different copulas viz. Gumbel-Hougaard and Bivariate Clayton.

(i) When Gumbel-Hougaard copula is applied to analyse the model then in the equations (15), (17), (21), (23), (30), (34) and (36), $\psi(y), \psi(z), \lambda$ and $\xi$ will have the following expressions:

$\psi(y)=\exp \left[-\left\{\left(-\log \psi_{P}(y)\right)^{\theta}+\left(-\log \phi_{P}(y)\right)^{\theta}\right\}^{1 / \theta}\right]$ $\psi(z)=\exp \left[-\left\{\left(-\log \psi_{c}(z)\right)^{\theta}+\left(-\log \phi_{c}(z)\right)^{\theta}\right\}^{1 / \theta}\right.$ $\lambda=\exp \left[-\left\{\left(-\log \lambda_{c}\right)^{\theta}+\left(-\log (\log z)^{\theta}\right\}^{1 / \theta}\right.\right.$ $\xi(y)=\phi_{P}(y)+\psi(y)$

(ii) When Bivariate-Clayton copula is applied to analyse the model then in the equations (15), (17), (21), (23), (30), (34) and (36), $\psi(\mathrm{y}), \psi(\mathrm{z}), \lambda$ and $\xi$ will have the following expressions:

$$
\begin{aligned}
& \psi(y)=\left[\left\{\psi_{P}(y)\right\}^{-\theta}+\left\{\phi_{P}(y)\right\}^{-\theta}\right]^{-1 / \theta} \\
& \psi(z)=\left[\left\{\psi_{c}(z)\right\}^{-\theta}+\left\{\phi_{c}(z)\right\}^{-\theta}\right]^{-1 / \theta} \\
& \lambda=\left[\left(\lambda_{c}\right)^{-\theta}+(\log z)^{-\theta}\right]^{-1 / \theta} \\
& \xi(y)=\phi_{P}(y)+\psi(y)
\end{aligned}
$$

\section{Asymptotic behaviour of the system}

Using Able's lemma

$$
\lim _{s \rightarrow 0}\{s \bar{F}(s)\}=\lim _{t \rightarrow \infty} F(t)=F(\text { say })
$$

In equations (37) and (38) we get the following time independent probabilities 
$\bar{P}_{\text {up }}(s)=\left\{1+2 \lambda_{p}\left[\frac{1-\bar{S}_{\phi_{p}}\left(s^{* *}\right)}{s * *}\right]\right\} \frac{1}{D(0)}$

$\bar{P}_{\text {down }}(s)=\frac{1}{D(0)}\left[\left[\lambda_{A} \bar{M}_{\phi_{A}}+\lambda \bar{M}_{\psi_{z}}\right]+2 \lambda_{A} \lambda_{P} \bar{M}_{\phi_{A}}\right.$

$\left\{\frac{1-\bar{S}_{\phi p}\left(s^{* *}\right)}{s * *}\right\}+\left[\frac{1-\bar{S}_{\phi p}\left(s^{* *}+\eta(u)\right)}{s^{* *}+\eta(u)}\right]$

$\left.2 \lambda_{P}^{2}+\eta(u) 2 \lambda_{P}^{2}\left[\frac{1-\bar{S}_{\xi}\left(s^{* * *}+\eta(u)\right)}{s^{* *}+\eta(u)}\right]\right]$

where

$D(0)=\lim _{s \rightarrow 0} D(s)$

$\bar{M}_{\phi_{A}}=\lim _{s \rightarrow 0}\left\{\frac{1-\bar{S}_{\phi_{A}}(s)}{s}\right\}$

$S^{* *}=\lim _{s \rightarrow 0} s^{*}$

The present model has been studied under two different copulas viz. Gumbel-Hougaard and Bivariate Clayton.

(i) When Gumbel-Hougaard copula is applied to analyse the model then in the equations (40), (41) and (42), $\psi(\mathrm{y}), \psi(\mathrm{z}), \lambda$ and $\xi$ will have the following expressions:

$\psi(y)=\exp \left[-\left\{\left(-\log \psi_{P}(y)\right)^{\theta}+\left(-\log \phi_{P}(y)\right)^{\theta}\right\}^{1 / \theta}\right]$

$\psi(z)=\exp \left[-\left\{\left(-\log \psi_{c}(z)\right)^{\theta}+\left(-\log \phi_{c}(z)\right)^{\theta}\right\}^{1 / \theta}\right.$

$\lambda=\exp \left[-\left\{\left(-\log \lambda_{c}\right)^{\theta}+\left(-\log (\log z)^{\theta}\right\}^{1 / \theta}\right.\right.$

$\xi(y)=\phi_{P}(y)+\psi(y)$

(ii) When Bivariate-Clayton copula is applied to analyse the model then in the equations (40), (41) and (42), $\psi(\mathrm{y}), \psi(\mathrm{z}), \lambda$ and $\xi$ will have the following expressions:

$\psi(y)=\left[\left\{\psi_{P}(y)\right\}^{-\theta}+\left\{\phi_{P}(y)\right\}^{-\theta}\right]^{-1 / \theta}$

$\psi(z)=\left[\left\{\psi_{c}(z)\right\}^{-\theta}+\left\{\phi_{c}(z)\right\}^{-\theta}\right]^{-1 / \theta}$

$\lambda=\left[\left(\lambda_{c}\right)^{-\theta}+(\log z)^{-\theta}\right]^{-1 / \theta}$

$\xi(y)=\phi_{P}(y)+\psi(y)$

\section{Particular cases}

(1) When catastrophic failure does not occur in the subsystem B.
In this by putting $\lambda_{C}=0$ in equations (37) and (38), we get

$$
\begin{aligned}
& \bar{P}_{\text {up }}(s)=\bar{P}_{0}(s)+\bar{P}_{3}(s) \\
& =\left\{1+2 \lambda_{P}\left[\frac{1-\bar{S}_{\phi_{P}}\left(s^{*}\right)}{s *}\right]\right\} \bar{P}_{0}(s) \\
& \bar{P}_{\text {down }}(s)=\bar{P}_{1}(s)+\bar{P}_{2}(s)+\bar{P}_{4}(s)+\bar{P}_{5}(s)+\bar{P}_{6}(s) \\
& =\lambda_{\text {A }}\left\{\frac{1-\bar{S}_{\phi_{A}}(s)}{s}\right\} \bar{P}_{0}(s)+2 \lambda_{A} \lambda_{P}\left\{\frac{1-\bar{S}_{\phi_{A}}(s)}{s}\right\} \times \\
& \left\{\frac{1-\bar{S}_{\phi_{P}}\left(s^{*}\right)}{s^{*}}\right\} \bar{P}_{0}(s)+2 \lambda_{P}{ }^{2} \bar{P}_{0}(s) \times \\
& {\left[\frac{1-\bar{S}_{\phi P}(s+s *+\eta(u))}{s+s^{*}+\eta(u)}\right]+2 \eta(u) \lambda_{P}{ }^{2} \bar{P}_{0}(s) \times} \\
& {\left[\frac{1-\bar{S}_{\xi}(2 s+s *+\eta(u))}{2 s+s^{*}+\eta(u)}\right]}
\end{aligned}
$$

where

$\bar{P}_{0}(s)=\frac{1}{D_{1}(s)}$

$D_{1}(s)=s+\lambda_{A}+2 \lambda_{P}-\lambda_{A} \bar{S}_{\phi_{A}}(s)-2 \lambda_{P} \bar{S}_{\phi_{P}}\left(s^{*}\right)$

$-2 \lambda_{P}{ }^{2} \eta(u) \times \int_{0}^{\infty} \psi(y) \exp \left\{-\left(2 s+s^{*}+\eta(u)\right) y\right.$

$\left.-\int_{0}^{y} \xi(y) d y\right\}$

$s^{*}=s+\lambda_{A}+\lambda_{P}-\lambda_{A} \bar{S}_{\phi_{A}}(s)$

The present model has been studied under two different copulas viz. Gumbel-Hougaard and Bivariate Clayton.

(i) When Gumbel-Hougaard copula is applied to analyse the model then in the equations (46) and (48), $\psi(\mathrm{y})$ and $\xi$ will have the following expressions:

$$
\begin{aligned}
& \psi(y)=\exp \left[-\left\{\left(-\log \psi_{P}(y)\right)^{\theta}+\left(-\log \phi_{P}(y)\right)^{\theta}\right\}^{1 / \theta}\right] \\
& \xi(y)=\phi_{P}(y)+\psi(y)
\end{aligned}
$$

(ii) When Bivariate-Clayton copula is applied to analyse the model then in the equations (46), (48), $\psi(\mathrm{y})$ and $\xi$ will have the following expressions:

$$
\begin{aligned}
& \psi(y)=\left[\left\{\psi_{p}(y)\right\}^{-\theta}+\left\{\phi_{p}(y)\right\}^{-\theta}\right]^{-1 / \theta} \\
& \xi(y)=\phi_{p}(y)+\psi(y)
\end{aligned}
$$

(2) When repair follows exponential distribution. 
In this case the result can be derived by putting

$\bar{S}_{\psi_{z}}(s)=\frac{\exp \left[-\left\{\left(-\log \psi_{c}(z)\right)^{\theta}+\left(-\log \phi_{c}(x)\right)^{\theta}\right\}^{1 / \theta}\right]}{s+\exp \left[-\left\{\left(-\log \psi_{c}(z)\right)^{\theta}+\left(-\log \phi_{c}(x)\right)^{\theta}\right\}^{1 / \theta}\right]}$

$\bar{S}_{\phi_{P}}(s)=\frac{\phi_{P}(y)}{s+\phi_{P}(y)}, \bar{S}_{\phi_{A}}(s)=\frac{\phi_{A}(x)}{s+\phi_{A}(x)}$

In equations (37) and (38), we get

$$
\begin{aligned}
& \bar{P}_{\text {up }}(s)=\bar{P}_{0}(s)+\bar{P}_{3}(s) \\
&=\left\{1+2 \lambda_{P}\left[\frac{1}{s *+\phi_{P}}\right]\right\} \bar{P}_{0}(s) \\
& \bar{P}_{\text {down }}(s)=\bar{P}_{1}(s)+\bar{P}_{2}(s)+\bar{P}_{4}(s)+\bar{P}_{s}(s)+\bar{P}_{6}(s) \\
&= {\left[\lambda_{\text {A }}\left\{\frac{1}{s+\phi_{A}}\right\}+\lambda\left\{\frac{1}{s+\psi_{2}}\right\}\right] \bar{P}_{0}(s) } \\
&+2 \lambda_{A} \lambda_{P}\left\{\frac{1}{s+\phi_{A}}\right\} \times\left\{\frac{1}{s^{*}+\phi_{P}}\right\} \bar{P}_{0}(s) \\
&+2 \lambda_{P}^{2} \bar{P}_{0}(s) \times\left[\frac{1}{s+s^{*}+\eta(u)+\phi_{P}}\right] \\
&+2 \eta(u) \lambda_{P}^{2} \bar{P}_{0}(s)\left[\frac{1}{2 s+s^{*}+\eta(u)+\xi}\right]
\end{aligned}
$$

where

$\bar{P}_{0}(s)=\frac{1}{D_{2}(s)}$

$D_{2}(s)=s+\lambda_{A}+\lambda+2 \lambda_{P}-\lambda_{A} \frac{\phi_{A}}{s+\phi_{A}}$

$-\lambda \frac{\psi(z)}{s+\psi(z)}-\frac{2 \lambda_{p} \phi_{p}}{s^{*}+\phi_{p}}-2 \lambda_{P}^{2} \eta(u) \times$

$\int_{0}^{\infty} \psi(y) \exp \left\{-\left(2 s+s^{*}+\eta(u)\right) y-\int_{0}^{y} \xi(y) d y\right\}$

The present model has been studied under two different copulas viz. Gumbel-Hougaard and Bivariate Clayton.

(i) When Gumbel-Hougaard copula is applied to analyse the model then in the equations (52) and (54), $\psi(\mathrm{y}), \psi(\mathrm{z}), \lambda$ and $\xi$ will have the following expressions:

$\psi(y)=\exp \left[-\left\{\left(-\log \psi_{P}(y)\right)^{\theta}+\left(-\log \phi_{P}(y)\right)^{\theta}\right\}^{1 / \theta}\right]$

$\psi(z)=\exp \left[-\left\{\left(-\log \psi_{c}(z)\right)^{\theta}+\left(-\log \phi_{c}(z)\right)^{\theta}\right\}^{1 / \theta}\right.$ $\lambda=\exp \left[-\left\{\left(-\log \lambda_{c}\right)^{\theta}+\left(-\log (\log z)^{\theta}\right\}^{1 / \theta}\right.\right.$

$\xi(y)=\phi_{P}(y)+\psi(y)$

(ii) When Bivariate-Clayton copula is applied to analyse the model then in the equations (52) and (54), $\psi(\mathrm{y}), \psi(\mathrm{z}), \lambda$ and $\xi$ will have the following expressions:

$$
\begin{aligned}
& \psi(y)=\left[\left\{\psi_{P}(y)\right\}^{-\theta}+\left\{\phi_{P}(y)\right\}^{-\theta}\right]^{-1 / \theta} \\
& \psi(z)=\left[\left\{\psi_{c}(z)\right\}^{-\theta}+\left\{\phi_{c}(z)\right\}^{-\theta}\right]^{-1 / \theta} \\
& \lambda=\left[\left(\lambda_{c}\right)^{-\theta}+(\log z)^{-\theta}\right]^{-1 / \theta} \\
& \xi(y)=\phi_{P}(y)+\psi(y)
\end{aligned}
$$

\section{Numerical computation}

\subsection{Availability analysis}

Let the repair follows exponential distribution. Also let vacation rate be $\eta(u)=0.5$, failure rates of subsystem $A$ and $B$ for partial and catastrophic failures be $\lambda_{\mathrm{A}}=0.5, \lambda_{\mathrm{P}}$ $=0.25, \lambda_{\mathrm{C}}=0.25$, repair rates be $\Phi_{\mathrm{P}}=\Phi_{\mathrm{C}}=\Phi_{\mathrm{A}}=\psi_{\mathrm{P}}=1$, $\theta=1$ and $\mathrm{x}=\mathrm{y}=\mathrm{z}=1$.

Putting all these values in equation (37), using equation (50) and taking inverse Laplace transformation, we get:

(i) In case of Gumbel-Hougaard copula:

$$
\begin{aligned}
\mathrm{P}_{\text {up }}= & -0.03612485642 \mathrm{e}^{(-2.510615836 \mathrm{t})}+0.3068736726 \\
& \mathrm{e}^{(-1.557348320 \mathrm{t})}+0.06456337718 \mathrm{e}^{(-1.425285235 \mathrm{t})} \\
& -0.001878777739 \mathrm{e}^{(-0.6777682208 \mathrm{t})}-0.005058707285 \\
& \mathrm{e}^{(-0.6247959226 \mathrm{t})}+0.6716252918 \mathrm{e}^{(-0.03751979833 \mathrm{t})}
\end{aligned}
$$

Table 2: Time vs. Availability for Gumbel-Hougaard copula

\begin{tabular}{cc}
\hline Time & $\mathrm{P}_{\text {up }}$ \\
\hline 0 & 1.000000 \\
1 & 0.720476 \\
2 & 0.638253 \\
3 & 0.602853 \\
4 & 0.578305 \\
5 & 0.556385 \\
6 & 0.536127 \\
7 & 0.516421 \\
8 & 0.497432 \\
9 & 0.479132 \\
10 & 0.464976 \\
\hline
\end{tabular}




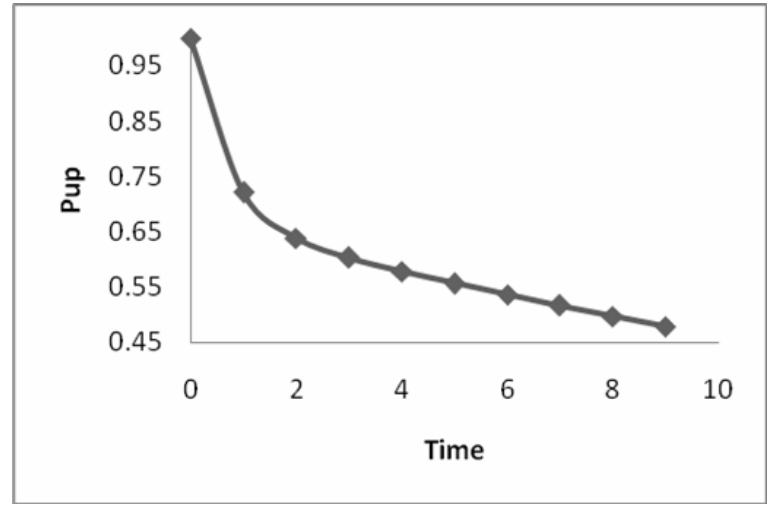

Fig. 3: Time vs. Availability for Gumbel-Hougaard copula

(ii) In case of Bivariate Clayton copula:

$$
\begin{aligned}
\mathrm{P}_{\text {up }}= & 0.06406442012 \mathrm{e}^{(-2.663714173 \mathrm{t})}+0.3687669327 \\
& \mathrm{e}^{(-1.748168308 \mathrm{t})}+0.007333724874 \mathrm{e}^{(-1.4444003723 \mathrm{t})}- \\
& 0.008933923194 \mathrm{e}^{(-0.6562343416 \mathrm{t})}-0.00857068425 \\
& \mathrm{e}^{(-0.6223021709 \mathrm{t})}+0.5773259140 \mathrm{e}^{(-0.03224395049 \mathrm{t})}
\end{aligned}
$$

Table 3: Time vs. Availability for Bivariate-Clayton copula

\begin{tabular}{cc}
\hline Time & $\mathrm{P}_{\text {up }}$ \\
\hline 0 & 1.000000 \\
1 & 0.620174 \\
2 & 0.548297 \\
3 & 0.523589 \\
4 & 0.506472 \\
5 & 0.490712 \\
6 & 0.475406 \\
7 & 0.460479 \\
8 & 0.445955 \\
9 & 0.431851 \\
10 & 0.418173 \\
\hline
\end{tabular}

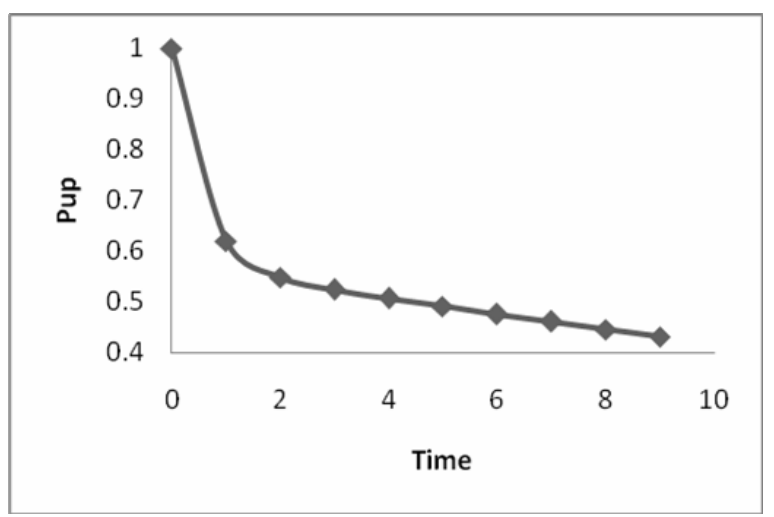

Fig. 4: Time vs. Availability for Bivariate-Clayton copula
Now in equations (55) and (56) setting $\mathrm{t}=0,1,2,3$, $4,5,6,7,8,9,10$, one can obtain Tables 2 and 3 respectively. Also Figs. 3 and 4 show the variation of availability with respect to time in these two cases respectively.

\subsection{Reliability Analysis}

For reliability analysis of the present model let the repair follows exponential distribution and $\lambda_{\mathrm{A}}=0.5, \lambda_{\mathrm{P}}=$ $0.25, \lambda_{\mathrm{C}}=0.25, \Phi_{\mathrm{P}}=\Phi_{\mathrm{C}}=\Phi_{\mathrm{A}}=\psi_{\mathrm{P}}=0, \theta=1$ and $\mathrm{x}=\mathrm{y}$ $=\mathrm{z}=1$.

Putting all these values in equation (37) and using (50), taking inverse Laplace transform and varying time, one can obtain Tables 4 and 5 and correspondingly Figs. 5 and 6 with respect to Gumbel-Hougaard and Bivariate Clayton copula respectively. The Tables and Figs. Are given below:

Table 4: Time vs. Reliability for Gumbel-Hougaard copula

\begin{tabular}{cc}
\hline Time & $\mathrm{P}_{\text {up }}$ \\
\hline 0 & 1.000000 \\
1 & 0.576854 \\
2 & 0.310925 \\
3 & 0.161011 \\
4 & 0.081258 \\
5 & 0.040298 \\
6 & 0.019739 \\
7 & 0.009583 \\
8 & 0.004622 \\
9 & 0.002218 \\
10 & 0.001061
\end{tabular}

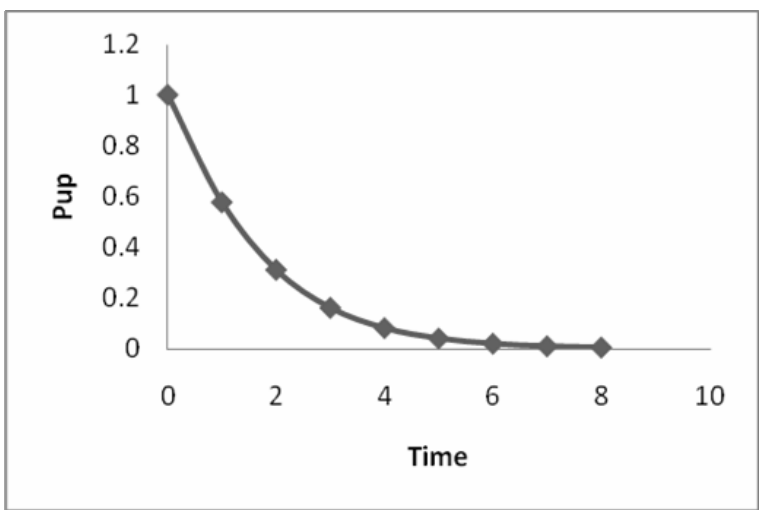

Fig. 5: Time vs. Reliability for Gumbel-Hougaard copula 
Table 5: Time vs. Reliability for Bivariate-Clayton copula

\begin{tabular}{cc}
\hline Time & $\mathrm{P}_{\text {up }}$ \\
\hline 0 & 1.000000 \\
1 & 0.442542 \\
2 & 0.209180 \\
3 & 0.092058 \\
4 & 0.043364 \\
5 & 0.020339 \\
6 & 0.009569 \\
7 & 0.004510 \\
8 & 0.002127 \\
9 & 0.001004 \\
10 & 0.000474 \\
\hline
\end{tabular}

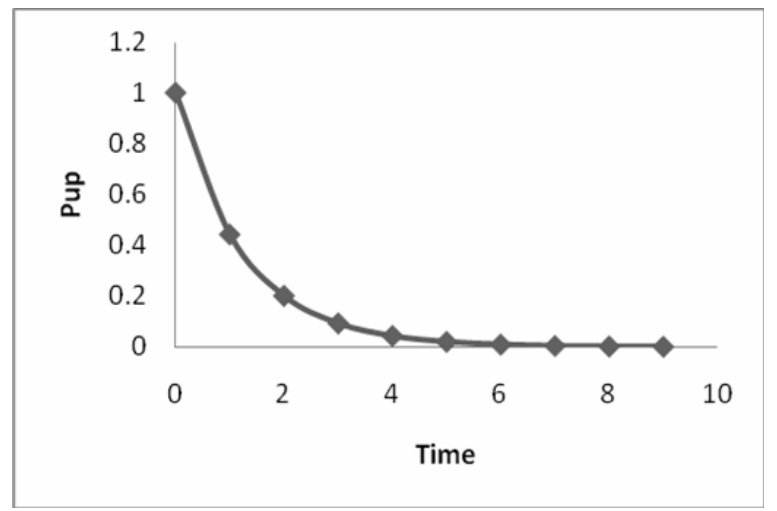

Fig. 6: Time vs. Reliability for Bivariate-Clayton copula

\subsection{T. T. F. Analysis}

M. T. T. F. of the system can be obtained as M. T. T. F. $=\lim _{s \rightarrow 0} \bar{P}_{u p}(s)$

Let repairs follow exponential distribution, then considering following cases, we can obtain the M. T. T. F. of the system with respect to different parameters. This can be obtained by substituting under mentioned values in the equation (50).

(a) Setting $\Phi_{\mathrm{A}}=\Phi_{\mathrm{C}}=\Phi_{\mathrm{P}}=\psi_{\mathrm{A}}=\psi_{\mathrm{P}}=0, \lambda_{\mathrm{P}}=0.15$, $\lambda_{\mathrm{C}}=0.10, \mathrm{x}=\mathrm{y}=\mathrm{z}=1, \theta=1$ and varying $\lambda_{\mathrm{A}}$ as 0.10 , $0.20,0.30,0.40,0.50,0.60,0.70,0.80,0.90$, one can obtain Tables 6 and 7 which show variation of M. T. T. F. with respect to $\lambda_{\mathrm{A}}$ in the case of Gumbel-Hougaard copula and Bivariate Clayton copula respectively.

(b) Setting $\Phi_{\mathrm{A}}=\Phi_{\mathrm{C}}=\Phi_{\mathrm{P}}=\psi_{\mathrm{A}}=\psi_{\mathrm{P}}=0, \lambda_{\mathrm{A}}=0.20$, $\lambda_{\mathrm{C}}=0.10, \mathrm{x}=\mathrm{y}=\mathrm{z}=1, \theta=1$ and varying $\lambda_{\mathrm{P}}$ as 0.10 , $0.20,0.30,0.40,0.50,0.60,0.70,0.80,0.90$, one can obtain Tables 8 and 9 which demonstrate variation of $\mathrm{M}$. T. T. F. with respect to $\lambda_{\mathrm{P}}$ in the case of GumbelHougaard copula and Bivariate Clayton copula respectively.

(c) Setting $\Phi_{\mathrm{A}}=\Phi_{\mathrm{C}}=\Phi_{\mathrm{P}}=\psi_{\mathrm{A}}=\psi_{\mathrm{P}}=0, \lambda_{\mathrm{A}}=0.20$, $\lambda_{\mathrm{P}}=0.15, \mathrm{x}=\mathrm{y}=\mathrm{z}=1, \theta=1$ and varying $\lambda_{\mathrm{C}}$ as 0.10 , $0.20,0.30,0.40,0.50,0.60,0.70,0.80,0.90$, one can obtain Tables 10 and 11 which show variation of M. T. T. F. with respect to $\lambda_{C}$ in the case of Gumbel-Hougaard and Bivariate Clayton copula respectively.

Variation of M. T. T. F with respect to $\lambda_{\mathrm{A}}, \lambda_{\mathrm{P}}$ and $\lambda_{\mathrm{C}}$ in the cases (a), (b) and (c) for Gumbel-Hougaard and Bivariate Clayton copula have been shown by the Figs: 7, 8, 9, 10, 11 and 12 respectively. The Figs. and Tables are given below:

Table 6: $\lambda_{\mathrm{A}}$ vs. M.T.T.F. for Gumbel-Hougaard copula

\begin{tabular}{cc}
\hline$\lambda_{\mathrm{A}}$ & MTTF \\
\hline .10 & 4.789117552 \\
.20 & 3.320033009 \\
.30 & 2.527646829 \\
.40 & 2.035166969 \\
.50 & 1.700699662 \\
.60 & 1.459283665 \\
.70 & 1.277112835 \\
.80 & 1.134912933 \\
.90 & 1.020914718
\end{tabular}

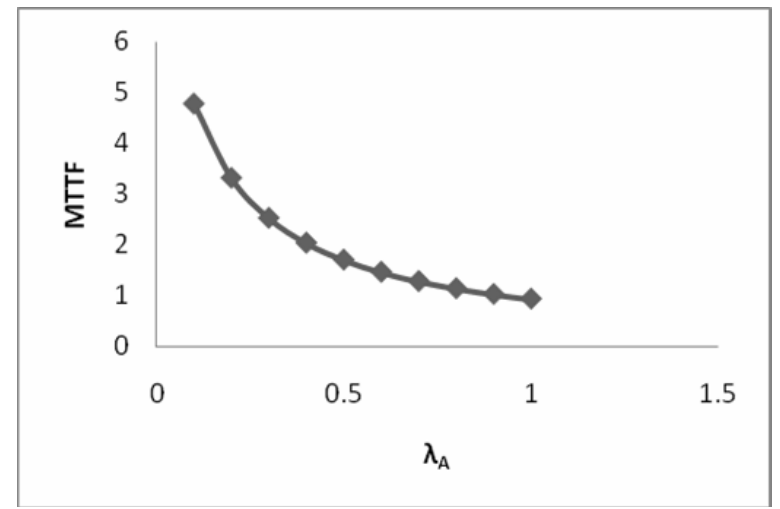

Fig. 7: $\lambda_{\mathrm{A}}$ vs. M.T.T.F. for Gumbel-Hougaard copula 
Table 7: $\lambda_{\mathrm{A}}$ vs. M.T.T.F. for Bivariate-Clayton copula

\begin{tabular}{cc}
\hline$\lambda_{\mathrm{A}}$ & MTTF \\
\hline .10 & 4.572318664 \\
.20 & 3.195599546 \\
.30 & 2.446819664 \\
.40 & 1.978419064 \\
.50 & 1.658659691 \\
.60 & 1.426887824 \\
.70 & 1.251383502 \\
.80 & 1.113984201 \\
.90 & 1.003557701 \\
\hline
\end{tabular}

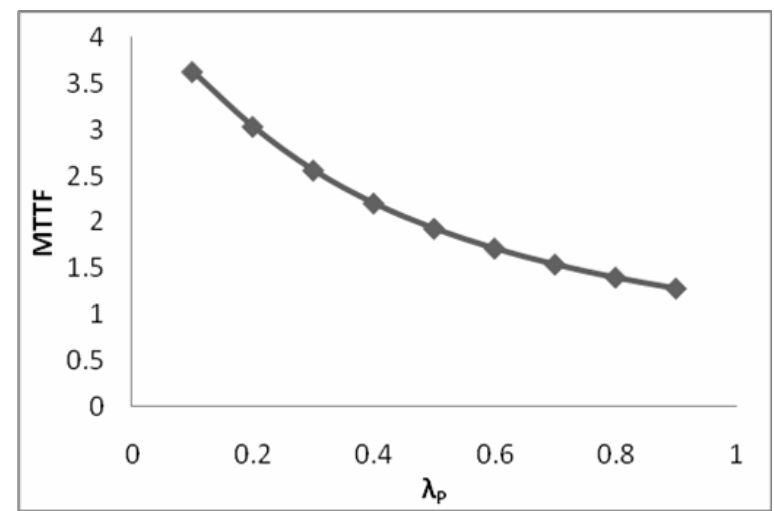

Fig. 9: $\lambda_{P}$ vs. M.T.T.F. for Gumbel-Hougaard copula

Table 9: $\lambda_{P}$ vs. M.T.T.F. for Bivariate-Clayton copula

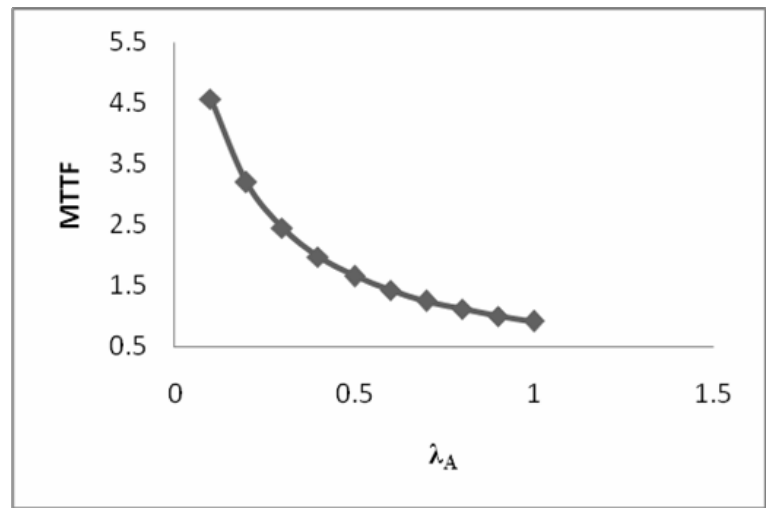

\begin{tabular}{cc}
\hline$\lambda_{\mathrm{P}}$ & MTTF \\
\hline .10 & 3.463877777 \\
.20 & 2.936183598 \\
.30 & 2.496719324 \\
.40 & 2.158183142 \\
.50 & 1.895608990 \\
.60 & 1.687870466 \\
.70 & 1.520117750 \\
.80 & 1.382128630 \\
.90 & 1.266778289 \\
\hline
\end{tabular}

Fig. 8: $\lambda_{\mathrm{A}}$ vs. M.T.T.F. for Bivariate-Clayton copula

Table 8: $\lambda_{P}$ vs. M.T.T.F. for Gumbel-Hougaard copula

\begin{tabular}{cc}
\hline$\lambda_{\mathrm{P}}$ & MTTF \\
\hline .10 & 3.628119357 \\
.20 & 3.033176195 \\
.30 & 2.560000544 \\
.40 & 2.202556920 \\
.50 & 1.928394467 \\
.60 & 1.713062314 \\
.70 & 1.540071314 \\
.80 & 1.398319465 \\
.90 & 1.280176690 \\
\hline
\end{tabular}

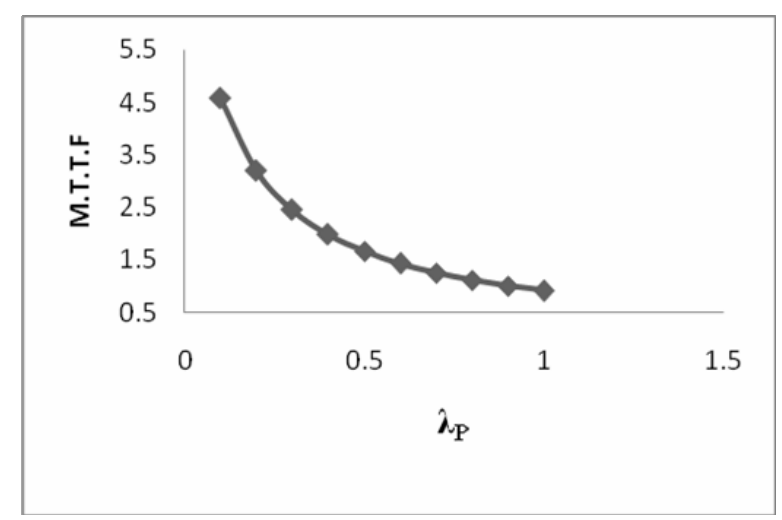

Fig. 10: $\lambda_{P}$ vs. M.T.T.F. for Bivariate-Clayton copula 
Table 10: $\lambda_{\mathrm{C}}$ vs. M.T.T.F. for Gumbel-Hougaard copula

\begin{tabular}{cc}
\hline$\lambda_{\mathrm{C}}$ & MTTF \\
\hline .10 & 3.320033009 \\
.20 & 3.001444774 \\
.30 & 2.738646057 \\
.40 & 2.518162301 \\
.50 & 2.330534884 \\
.60 & 2.168928741 \\
.70 & 2.028281721 \\
.80 & 1.904764759 \\
.90 & 1.795427972 \\
\hline
\end{tabular}

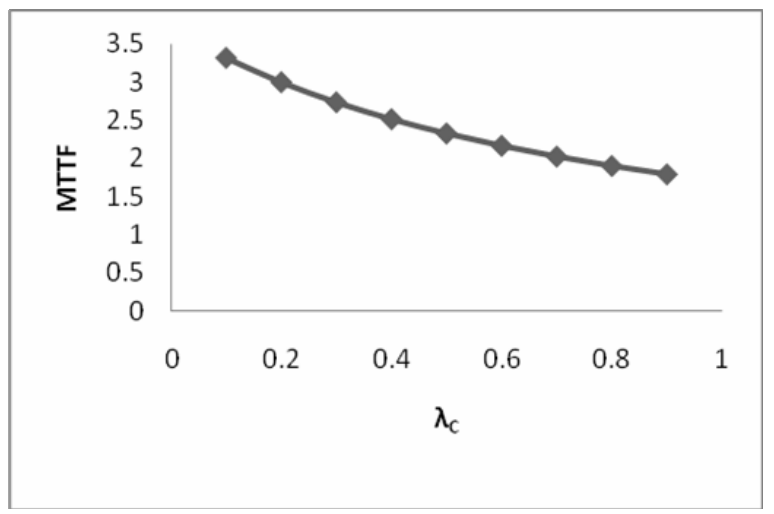

Fig. 11: $\lambda_{C}$ vs. M.T.T.F. for Gumbel-Hougaard copula

Table 11: $\lambda_{\mathrm{C}}$ vs. M.T.T.F. for Bivariate-Clayton copula

\begin{tabular}{cc}
\hline$\lambda_{\mathrm{C}}$ & MTTF \\
\hline .10 & 3.195599546 \\
.20 & 2.917391153 \\
.30 & 2.743894466 \\
.40 & 2.625355735 \\
.50 & 2.539232103 \\
.60 & 2.473826713 \\
.70 & 2.422466162 \\
.80 & 2.381065192 \\
.90 & 2.346982891 \\
\hline
\end{tabular}

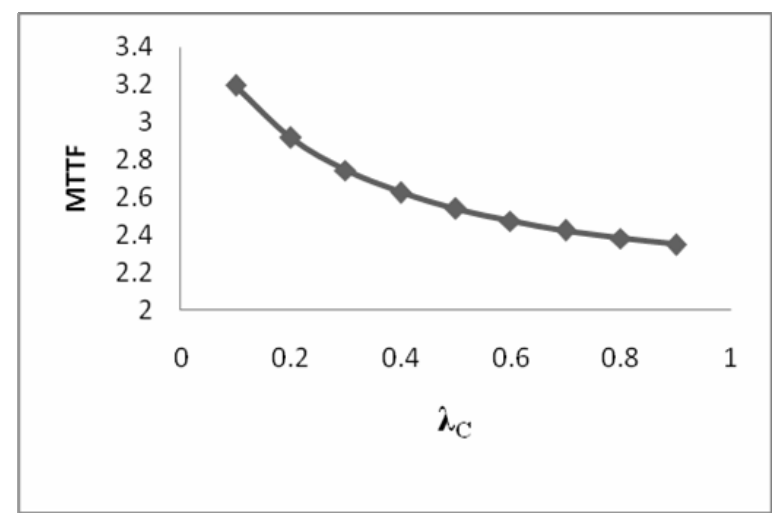

Fig. 12: $\lambda_{\mathrm{C}}$ vs. M.T.T.F. for Bivariate-Clayton copula

\subsection{Cost Analysis}

Letting vacation rate to be $\eta(\mathrm{u})=0.5$, failure rates of subsystem $A$ and $B$ for partial and catastrophic failures be $\lambda_{\mathrm{A}}=0.5, \lambda_{\mathrm{P}}=0.25, \lambda_{\mathrm{C}}=0.25$, repair rates be $\Phi_{\mathrm{P}}=\Phi_{\mathrm{C}}$ $=\Phi_{\mathrm{A}}=\psi_{\mathrm{P}}=1$ and $\mathrm{x}=\mathrm{y}=\mathrm{z}=1$. Furthermore, if the repair follows exponential distribution then from equation (50), on putting all these values and taking inverse Laplace transform one can obtain equations (55) and (56). If the service facility is always available, then expected profit during the interval $(0, t]$ is given by

$$
E_{P}(t)=K_{1} \int_{0}^{t} P_{u p}(t) d t-K_{2} t
$$

where $\mathrm{K}_{1}$ and $\mathrm{K}_{2}$ are the revenue per unit time and service cost per unit time respectively,

(i) In case of Gumbel-Hougaard copula:

$E_{P}(t)=K_{1}\left[0.001438884273 e^{(-2.510615836 t)}\right.$

$$
\begin{aligned}
& -.1970488353 \mathrm{e}^{(-1.557348320 \mathrm{t})}-0.04529856593 \\
& \mathrm{e}^{(-1.425285235 \mathrm{t})}+0.002772006242 \mathrm{e}^{(-0.6777682208 \mathrm{t})}+ \\
& 0.008096575381 \mathrm{e}^{(-0.6247959226 \mathrm{t})}-17.90055708 \\
& \left.\mathrm{e}^{(-.035751979833 \mathrm{t})}+18.11764706\right]-\mathrm{K}_{2} \mathrm{t}
\end{aligned}
$$

(ii) In case of Bivariate Clayton copula:

$$
\begin{aligned}
E_{P}(t)= & K_{1}\left[-0.02405078622 \mathrm{e}^{(-2.663714173 t)}\right. \\
& -.2109447534 \mathrm{e}^{(-1.748168308 \mathrm{t})}-0.005078743744 \\
& \mathrm{e}^{(-1.444003723 \mathrm{t})}+0.01361392208 \mathrm{e}^{(-0.6562343416 \mathrm{t})}+ \\
& 0.01375066459 \mathrm{e}^{(-0.6223021709 \mathrm{t})}-17.90493737 \\
& \left.\mathrm{e}^{(-.03224395049 \mathrm{t})}+18.11764707\right]-\mathrm{K}_{2} \mathrm{t}
\end{aligned}
$$

Keeping $\mathrm{K}_{1}=1$ and varying $\mathrm{K}_{2}$ at $0.1,0.2,0.3,0.4,0.5$ in equations (57) and (58), one can obtain Tables 12 and 13 which is depicted by Figs. 13 and 14 respectively. 
Analysis of a Risky Two Unit System under Marked Process Incorporating Two Repairmen with Vacations Table 12: Time vs. expected profit for Gumbel-Hougaard copula

\begin{tabular}{cccccc}
\hline Time & \multicolumn{5}{c}{$\mathrm{E}_{\mathrm{P}}(\mathrm{t})$} \\
\hline & $\mathrm{K}_{2}=0.1$ & $\mathrm{~K}_{2}=0.2$ & $\mathrm{~K}_{2}=0.3$ & $\mathrm{~K}_{2}=0.4$ & $\mathrm{~K}_{2}=0.5$ \\
0 & 0 & 0 & 0 & 0 & 0 \\
1 & 0.730773 & 0.630773 & 0.530773 & 0.430773 & 0.330773 \\
2 & 1.302943 & 1.102943 & 0.902943 & 0.702943 & 0.502943 \\
3 & 1.838773 & 1.538773 & 1.238773 & 0.938773 & 0.638773 \\
4 & 2.312025 & 1.912025 & 1.512025 & 1.112025 & 0.712025 \\
5 & 2.779364 & 2.779364 & 1.779364 & 1.279364 & 0.779364 \\
6 & 3.225671 & 2.625671 & 2.025671 & 1.425671 & 0.825671 \\
7 & 3.651885 & 2.951885 & 2.251885 & 1.551885 & 0.851885 \\
8 & 4.058754 & 3.258754 & 2.458754 & 1.658754 & 0.858754 \\
9 & 4.446980 & 3.546980 & 2.646980 & 1.746980 & 0.846980 \\
10 & 4.817240 & 3.817240 & 2.817240 & 1.817240 & 0.817240 \\
\hline
\end{tabular}

Table 13: Time vs. expected profit for Bivariate-Clayton copula

\begin{tabular}{cccccc}
\hline Time & \multicolumn{5}{c}{$\mathrm{E}_{\mathrm{P}}(\mathrm{t})$} \\
\hline & $\mathrm{K}_{2}=0.1$ & $\mathrm{~K}_{2}=0.2$ & $\mathrm{~K}_{2}=0.3$ & $\mathrm{~K}_{2}=0.4$ & $\mathrm{~K}_{2}=0.5$ \\
0 & 0 & 0 & 0 & 0 & 0 \\
1 & 0.655671 & 0.555671 & 0.455671 & 0.355671 & 0.255671 \\
2 & 1.131750 & 0.931750 & 0.731750 & 0.531750 & 0.331750 \\
3 & 1.566394 & 1.266394 & 0.966394 & 0.666394 & 0.366394 \\
4 & 1.981209 & 1.581209 & 1.181209 & 0.781209 & 0.381209 \\
5 & 2.379751 & 1.879751 & 1.379751 & 0.879751 & 0.379751 \\
6 & 2.762780 & 2.162780 & 1.562780 & 0.962780 & 0.362780 \\
7 & 3.130691 & 2.430691 & 1.730691 & 1.030691 & 0.330691 \\
8 & 3.483874 & 2.683874 & 1.883874 & 1.083874 & 0.283874 \\
9 & 3.822742 & 2.922742 & 2.022742 & 1.122742 & 0.222742 \\
10 & 4.147719 & 3.147719 & 2.147719 & 1.147719 & 0.147719 \\
\hline
\end{tabular}

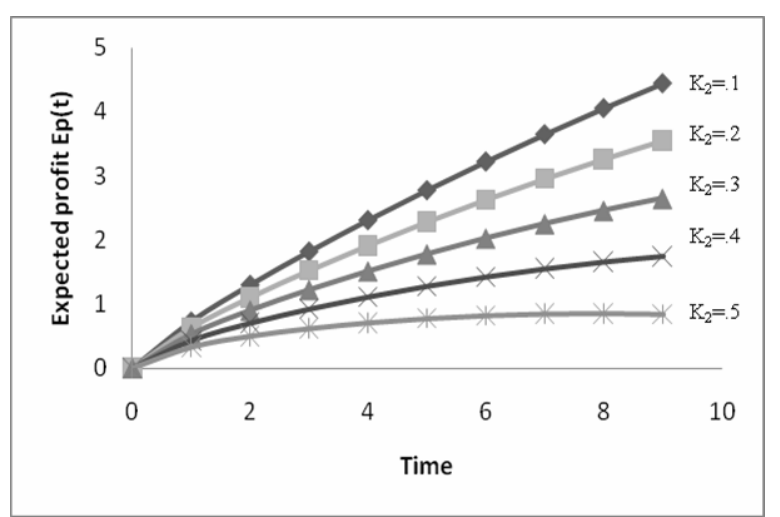

Fig. 13: Time vs. expected profit for Gumbel-Hougaard copula

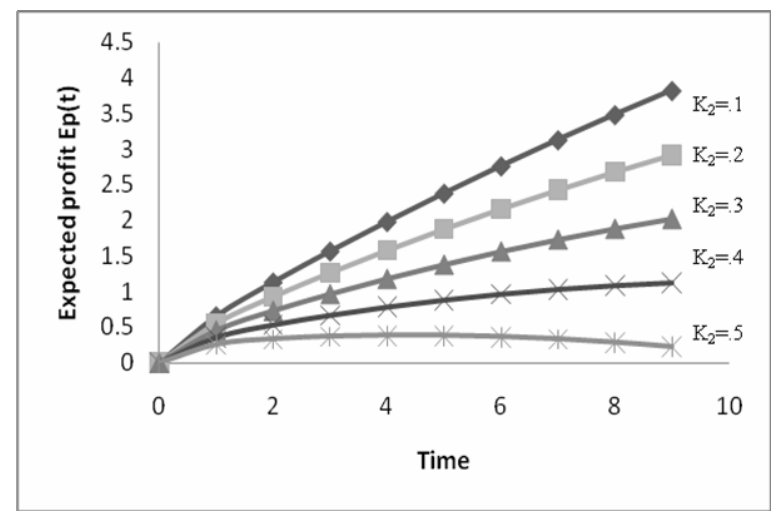

Fig. 14: Time vs. expected profit for Bivariate-Clayton copula 
10. Comparison of effect of head of line repair policy and preemptive-resume repair policy on the availability of the system

Let us take $\eta(\mathrm{u})=0.5, \lambda_{\mathrm{A}}=0.5, \lambda_{\mathrm{P}}=0.25$ and $\lambda_{\mathrm{C}}=0.25$, $\Phi_{\mathrm{P}}=\Phi_{\mathrm{C}}=\Phi_{\mathrm{A}}=\psi_{\mathrm{P}}=1, \theta=1$ and $\mathrm{x}=\mathrm{y}=\mathrm{z}=1$. Also let the repair follows exponential distribution.

Using all the above values in equation (50) and modelling the system for head of line repair policy the availability of the system is obtained as

$\mathrm{P}_{\text {up }}=-0.06521710750 \mathrm{e}^{(-2.380129270 \mathrm{t})}+0.3245962455$

$\mathrm{e}^{(-1.712176291 \mathrm{t})} \cos (0.3939583835 \mathrm{t})+0.1295409494$

$\mathrm{e}^{(-1.172176291 \mathrm{t})} \sin (0.3939583835)+$

$0.00001576068496 \mathrm{e}^{(-1.083764259 \mathrm{t})}+0.6101708864$

$\mathrm{e}^{(-0.02508722247 \mathrm{t})}$

Also from equation (55) the expression for the availability of the system in the case of preemptive resume repair policy is obtained as

$\mathrm{P}_{\text {up }}=-0.03612485642 \mathrm{e}^{(-2.510615836 \mathrm{t})}+0.3068736726$

$\mathrm{e}^{(-1.557348320 \mathrm{t})}+0.06456337718 \mathrm{e}^{(-1.425285235 \mathrm{t})}-$

$0.001878777739 \mathrm{e}^{(-0.6777682208 \mathrm{t})}-0.005058707285$

$\mathrm{e}^{(-0.6247959226 \mathrm{t})}+0.6716252918 \mathrm{e}^{(-0.03751979833 \mathrm{t})}$

which further yield the following Table.

Table 14: Availability vs time in two different repair disciplines

Time dependent up and down state probabilities

Preemptive- resume Head of line repair

repair

\begin{tabular}{|c|c|c|c|c|c|}
\hline S. & $\mathrm{Ti}$ & $P_{\text {up }}$ & $\mathrm{P}_{\text {down }}$ & $\mathrm{P}_{\text {up }}$ & $\mathrm{P}_{\text {down }}$ \\
\hline $\mathrm{N}$ & me & & & & \\
\hline \multicolumn{6}{|l|}{ o. } \\
\hline 1 & 0 & 1.000000 & 0 & 1.000000 & 0 \\
\hline 2 & 1 & 0.720476 & 0.279524 & 0.709318 & 0.290682 \\
\hline 3 & 2 & 0.638253 & 0.361747 & 0.611637 & 0.388363 \\
\hline 4 & 3 & 0.602853 & 0.397147 & 0.573202 & 0.426798 \\
\hline 5 & 4 & 0.578305 & 0.421695 & 0.553094 & 0.446906 \\
\hline 6 & 5 & 0.556635 & 0.443365 & 0.538220 & 0.461780 \\
\hline
\end{tabular}

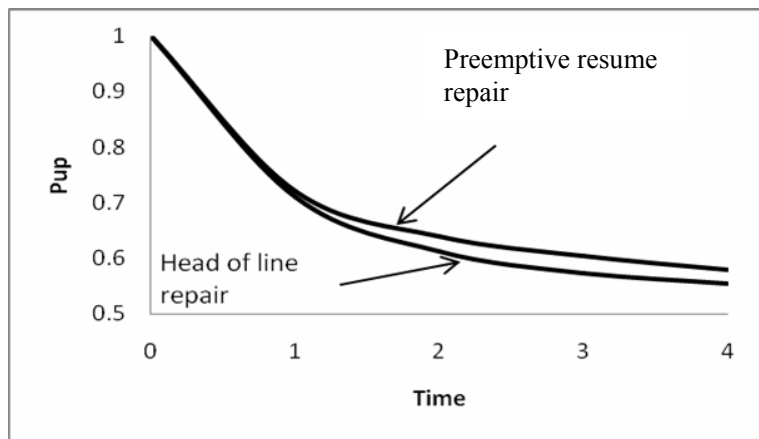

Fig. 15: Availability vs time in two repair disciplines
The Table 14 and correspondingly Fig. 15 represent the variation of availability with respect to time in two repair policies viz. "Head of line repair policy" and "Preemptive-resume repair policy". We find that in both the repair policies it decreases as the time increases. It can easily be concluded that the availability in preemptive-resume repair discipline is higher than the head of line repair discipline.

Hence preemptive resume repair policy should be adopted for repair purposes to obtain optimum returns wherever applicable.

\section{Comparison of effect of Gumbel-Hougaard and Bivariate Clayton copula on different measures of the system}

From Tables 2 and 3 one can easily conclude that the value of availability of the system in both the cases is initially same and decreases as time increases but availability in case of Gumbel-Hougaard copula is greater than that of Bivariate Clayton copula. Further on comparing reliability of the system given in Tables 4 and 5 we observe that reliability of the system also decreases with respect to time but again its value is high for Gumbel-Hougaard copula.

A critical examination of Tables 6, 7, 8, 9, 10 and 11 reveal that the M. T. T. F. in case of Gumbel-Hougaard copula varies from value 4.789-1.020, 3.628-1.280 and $3.320-1.795$ with respect to $\lambda_{\mathrm{A}}, \lambda_{\mathrm{P}}$ and $\lambda_{\mathrm{C}}$ respectively as time passes. While in case of Bivariate Clayton copula it changes from 4.572 to 1.003 , from 3.463 to 1.266 and from 3.195 to 2.346 with respect to $\lambda_{\mathrm{A}}, \lambda_{\mathrm{P}}$ and $\lambda_{\mathrm{C}}$ respectively with the increment in time. These Tables reveal that initially in each case M. T. T. F. is higher for Gumbel-Hougaard copula in comparison to Bivariate Clayton copula. But its decrement is more rapid in case of Gumbel-Hougaard copula than in case of Bivariate Clayton copula. Finally if we critically examine the expected profit of the system we find from Tables 12 and 13 that the maximum and minimum values of expected profit in case of Gumbel-Hougaard copula are 4.8172 and 0.3307 respectively. While in case of Bivariate Clayton copula these values are 4.1477 and 0.1477 respectively. So we can say that the profit will be large if we use Gumbel-Hougaard copula in place of Bivariate Clayton copula.

From the above study one can conclude that Gumbel-Hougaard copula is better than Bivariate Clayton copula in practical situations. 


\section{Discussion}

Several conclusions may be drawn on the basis of work and results presented in this paper.

(1) By the examination of Figs. 3, 4, 5 and 6 one can say that when $\eta(\mathrm{u})=0.5, \lambda_{\mathrm{A}}=0.5, \lambda_{\mathrm{P}}=0.25$ and $\lambda_{\mathrm{C}}$ $=0.25$, then availability and reliability of the system decreases as the time increases.

(2) From Figs. 13 and 14 one can easily observe that increasing service cost leads decrement in expected profit.

(3) The observation of Figs. 7, 8, 9, 10, 11 and 12 reveal that M.T.T.F. of the complex system decreases as the value of $\lambda_{A}, \lambda_{P}$ and $\lambda_{C}$ increases. Computation also shows that M.T.T.F. depends more on $\lambda_{\mathrm{A}}$ in comparison to $\lambda_{\mathrm{P}}$ and $\lambda_{\mathrm{C}}$.

(4) The comparison of two repair facilities shows that preemptive resume repair policy is better than head of line repair policy for the considered system.

(5) Comparison of two types of copula viz. GumbelHougaard copula and Bivariate Clayton copula reveal that Gumbel-Hougaard copula should be adopted instead of Bivariate Clayton copula for better results.

\section{References}

Bo H Lindqvist and Tim Bedford, The identifiability problem for repairable systems subject to competing risks, Applied probability trust. 36(3) (2004) 774-790.

P. P. Gupta and S. C. Agarwal, A parallel redundant complex system with two types of failure under preemptive-repeat repair discipline, Microelectron. Reliab. 24(3) (1984) 395399.

M. Ram and S. B. Singh, Availability, M.T.T.F. and cost analysis of complex system under preemptive repeat repair discipline using Gumbel-Hougaard family copula, International journal of Quality and Reliability management (IJQRM). 27(3) (2010) 376-395.

R. C. Garg and L. R. Goel, Cost analysis of a system with common cause failure and two types of repair facilities. Microelectron. Reliab. 25(2) (1985) 281-284.

P. P. Gupta and M. K. Sharma, Reliability and M.T.T.F evaluation of a two duplex-unit standby system with two types of repair, Microelectron. Reliab. 33(3) (1993) 291295.

L. R. Goel and R. Gupta, Profit analysis of a cold standby system with two repair distributions, Microelectron. Reliab. 25(3) (1985) 467-472.
Hu Linmin and Li Jiandong, Reliability analysis of a three unit system with vacation and priority, ICIC express Letters. 3(2) (2009) 171-176.

R. K. Tuteja and S. C. Malik, Reliability and profit analysis of two single unit models with three modes and different repair policies of repairmen who appear and disappear randomly, Microelectron. Reliab, 32(3) (1992) 351-356.

A. I. Kovalenko and V.P. Smolich, Reliability analysis for a two element system attended by two repairmen, Journal of Mathematical Sciences. 107(6) (2001) 4443-4447.

N. Tiwari, S. B. Singh, M. Ram, Reliability analysis of a complex system, attended by two repairmen with vacation under marked process with the application of copula, International Journal of Reliability and Applications. 11(2) (2010)107-122.

N. Tiwari, Suraj Bhan Singh, Analysis of a Complex System Modelled by a Marked Point Process and Assuming Vacations for a Repairman, Economic Quality Control. 25 (2) (2010) 221-242.

M. A. El-Damcese, Analysis of warm standby systems subject to common-cause failures with time varying failure and repair rates. Applied Mathematical Sciences. 3 (18) (2009) 853-860.

A. Kumar and S. B. Singh, Reliability analysis of an $n$ unit parallel standby system under imperfect switching using copula. Computer Modelling and New Technologies. 12(1) (2008) 47-55.

R. B. Nelson, An Introduction to Copulas, $2^{\text {nd }}$ edn (Springer, Newyork 2006).

Yan Jun, Enjoy the joy of copula. Journal of statistical software. 21(4) (2007) 1-21. 